\title{
Immunoprophylaxie des infections respiratoires
}

> L'immunothérapie anti-infectieuse par anticorps suscite un regain d'intérêt en raison de l'augmentation de la résistance aux antibiotiques et de l'absence de thérapies contre la majorité des infections virales. Les immunoglobulines intraveineuses (IVIG), par leur large spectre d'activité, les IVIG hyperimmunes, en raison de leur forte concentration en anticorps spécifiques, et les anticorps monoclonaux qui reconnaissent un épitope sélectionné, sont capables de neutraliser de nombreux agents infectieux. L'immunothérapie des infections respiratoires utilise la capacité des anticorps de neutraliser les micro-organismes inhalés et leurs produits cytopathogènes. Leur administration préventive (immunoprophylaxie) s'avère beaucoup plus efficace que leur administration curative, par le fait qu'elle inhibe les étapes précoces du processus infectieux. L'efficacité des anticorps administrés par voie topique, qui requiert des doses inférieures à celles de la voie systémique, et les nouvelles technologies de fabrication d'anticorps monoclonaux humanisés, offrent de réelles perspectives dans le traitement préventif et curatif des maladies infectieuses - en particulier respiratoires - actuelles ou émergentes. <
Christelle Dreffier, Françoise Ramisse, Jean-Michel Alonso

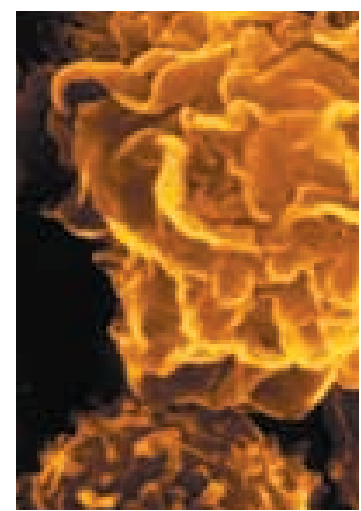

sérothérapie s'avérait efficace contre la diphtérie et le tétanos (elle en constitue d'ailleurs toujours le traitement), la pneumonie à pneumocoque, les méningites à méningocoques et à Hæmophilus influenzae, l'érysipèle et la scarlatine, et même certaines infections virales comme la rougeole ou la varicelle [2]. Si la sérothérapie constitue un traitement efficace des infections dans lesquelles la production d'une toxine est l'effecteur majeur de la pathogénicité (tétanos, diphtérie, botulisme), ses effets contre des agents infectieux exprimant des déterminants antigéniques variables sont dépendants de la présence d'anticorps reconnaissant spécifiquement le type antigénique du pathogène en cause [2]. Les antibiotiques, par leur large spectre d'activité, leur bonne tolérance et leur moindre coût, ont supplanté la sérothérapie dans les infections bactériennes, d'autant que l'utilisation de sérums animaux ou de préparations de sérums humains mal purifiées provoquait des réactions allergiques et inflammatoires. L'apparition croissante de bactéries résistantes à l'antibiothérapie [3], menaçant de recréer les dangers que présentaient les infections bactériennes durant l'ère pré-antibiotique, ainsi que l'absence de thérapies dans la majorité des infections virales, ont conduit à un regain d'intérêt pour l'immu- 
nothérapie par anticorps des maladies infectieuses [2]. Les progrès dans la purification des immunoglobulines plasmatiques ont permis l'élaboration de préparations injectables par voie veineuse bien tolérées et efficacement utilisées, par exemple, dans le traitement supplétif de nombreux déficits immunitaires $[4,5]$. Cet article expose les progrès thérapeutiques en matière d'immunoprophylaxie des maladies respiratoires apportés par les immunoglobulines intraveineuses (IVIG), l'immunothérapie par voie topique ou l'utilisation des anticorps monoclonaux.

\section{Problématique des infections respiratoires}

Les infections respiratoires sont une cause majeure de morbidité et de mortalité dans le monde, particulièrement chez les enfants et les patients atteints de déficits immunitaires [6]. Elles représentent une des indications majeures de l'antibiothérapie et ce sont parmi les agents bactériens responsables de ces infections (Streptococcus pneumoniae, Hæmophilus influenzae, Staphylococcus aureus, Branhamella catarrhalis) que sont apparus des variants polyrésistants en nombre croissant [3].

Les épithéliums respiratoires constituent un site primaire exposé au déclenchement d'infections potentiellement invasives. À la surface des épithéliums respiratoires, en complément des mécanismes de l'immunité innée (sécrétions microbicides, piégeage des particules et évacuation par battements ciliaires), les anticorps locaux ( $\operatorname{gg}$ et $\lg G$ ) constituent une première barrière efficace s'opposant à l'implantation, la prolifération et la dissémination des micro-organismes [7] (Figure 1). Dans de nombreuses conditions - physiologiques (immaturité immunitaire du jeune enfant, altérations immunitaires du sujet âgé), pathologiques (agressions atmosphériques, déficits immunitaires des maladies systémiques et déficits immunitaires viro-induits) ou iatrogènes (thérapeutiques invasives et immunosuppressives) -, la production de ces anticorps est déficiente ou altérée. Ces conditions prédisposent à des infections variées dont le diagnostic étiologique est rendu difficile par leur caractère atypique. L'immunothérapie par anticorps des infections respiratoires vise donc à restaurer la capacité des immunoglobulines de neutraliser les micro-organismes inhalés et leurs produits cytopathogènes.

\section{Immunothérapie passive des infections respiratoires par immunoglobulines humaines polyvalentes}

Les IVIG, purifiées et conditionnées industriellement par une dizaine de compagnies internationales, sont des préparations monomériques standardisées ( $98 \%$
d'IgG, incluant des IgGl, IgG2 et IgG3 dans les proportions normales du sérum [8]), dûment contrôlées pour l'absence d'agents pathogènes et dont l'efficacité a été démontrée dans de nombreuses études cliniques [9-15] récapitulées dans le Tableau I. Leur efficacité contre un large éventail d'infections est attribuée au fait qu'elles comportent une grande diversité d'anticorps contenus dans les plasmas des donneurs naturellement immunisés ou vaccinés contre une grande diversité de pathogènes primaires ou opportunistes [8]. Cependant, les effets observés dans certains essais cliniques sont parfois discordants $[10,11]$ (Tableau I), car les IVIG sont des mélanges d'IgG provenant des plasmas de milliers

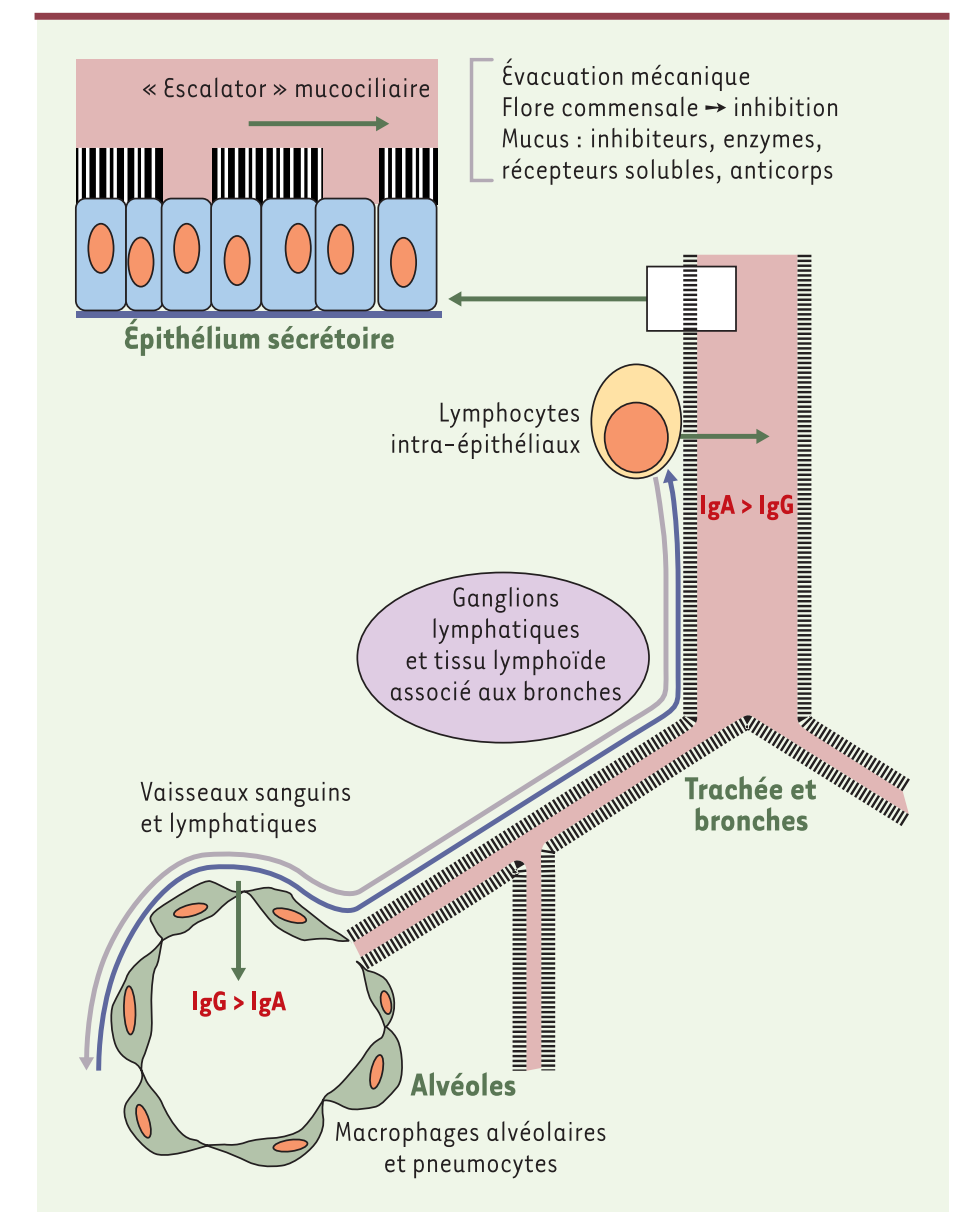

Figure 1. Bases de l'immunité des épithéliums respiratoires. L'immunité des épithéliums respiratoires repose sur les mécanismes de l'immunité innée déterminés principalement par l'«escalator» mucociliaire et la présence de sécrétions muqueuses et d'anticorps locaux. La flore commensale inhibe l'implantation de pathogènes exogènes. Le mucus contient des substances ayant des activités antimicrobiennes. Les immunoglobulines A sécrétoires (IgA) sont prédominantes dans le tractus respiratoire supérieur (du rhinopharynx aux bronchioles), tandis que, dans les alvéoles pulmonaires, ce sont essentiellement des IgG qui prédominent. Les IgA sont sécrétées dans les muqueuses par les lymphocytes $B$ intra-épithéliaux. Les IgG proviennent du plasma par extravasation. 
de donneurs et leur composition peut varier d'un fabricant à l'autre et de lot à lot [8]. De plus, la proportion d'anticorps spécifiques d'un type antigénique d'un pathogène que l'on veut neutraliser peut ne pas excéder $1 \%$ du titre en IgG d'une préparation d'IVIG [16]. Ces aléas dans la composition des IVIG sont en partie corrigés dans les préparations d'IVIG hyperimmunes obtenues à partir de donneurs dont les sérums ont un titre élevé d'anticorps contre un pathogène considéré.

\section{Immunothérapie des infections respiratoires par IVIG hyperimmunes}

Les IVIG hyperimmunes sont obtenues à partir du plasma de donneurs porteurs d'anticorps spécifiques ayant des titres élevés du fait d'une immunisation spontanée ou après vaccination. Les titres d'anticorps spécifiques peuvent être 5 à 10 fois plus élevés que dans les IVIG standard. Plusieurs essais cliniques démontrent l'efficacité de telles préparations (Tableaul). Mais, d'un point de vue pharmacocinétique, I'injection intraveineuse ne garantit pas la distribution des IgG du compartiment sanguin vers les épithéliums muqueux, sites primaires d'acquisition de la plupart des infections respiratoires. En effet, les IgG monomériques sont capturées de manière non spécifique par des récepteurs membranaires pour le fragment

\begin{tabular}{|c|c|c|}
\hline Maladies & Études & Références \\
\hline Infections nosocomiales chez les prématurés & $\begin{array}{l}\text { IVIG prophylactiques: } \\
\text { succès }(n=588) \\
\text { échec }(n=2416)\end{array}$ & $\begin{array}{l}{[10]} \\
{[11]}\end{array}$ \\
\hline $\begin{array}{l}\text { Infections opportunistes chez l'enfant } \\
\text { infecté par le VIH }\end{array}$ & $\begin{array}{l}\text { IVIG prophylactiques: } \\
\text { prévention des infections bactériennes } \\
\text { dans le groupe traité }\end{array}$ & {$[12]$} \\
\hline $\begin{array}{l}\text { Pneumonies chez des patients atteints } \\
\text { de déficit immunitaire } \\
\text { commun variable }\end{array}$ & $\begin{array}{l}\text { IVIG prophylactiques: diminution } \\
\text { du nombre de pneumonies }\end{array}$ & {$[4]$} \\
\hline Infections chez les polytraumatisés & $\begin{array}{l}\text { Prophylaxie par IVIG }(n=21) \text { ou albumine } \\
(n=18) \text { : augmentation de la bactéricidie } \\
\text { du sérum chez les patients traités par IVIG }\end{array}$ & {$[13]$} \\
\hline $\begin{array}{l}\text { Pneumopathie à cytomégalovirus } \\
\text { chez le transplanté rénal }\end{array}$ & $\begin{array}{l}\text { IVIG hyperimmunes comparées } \\
\text { au ganciclovir: efficacité égale }\end{array}$ & {$[14]$} \\
\hline Bronchiolite à virus respiratoire syncytial & $\begin{array}{l}\text { Essai préventif chez les enfants à risque } \\
\text { par IVIG hyperimmunes: protection } \\
\text { à haute dose }(750 \mathrm{mg} / \mathrm{kg})\end{array}$ & [9] \\
\hline $\begin{array}{l}\text { Coqueluche à } B \text {. pertussis chez des enfants } \\
\text { de moins de } 3 \text { ans }\end{array}$ & $\begin{array}{l}\text { Essai curatif par injection d'IVIG } \\
\text { hyperimmunes }(n=33) \\
\text { ou d'albumine }(n=14) \text { : } \\
\text { amélioration clinique dans le groupe IVIG }\end{array}$ & {$[15]$} \\
\hline
\end{tabular}

Tableau I. Quelques exemples d'essais cliniques d'immunoprophylaxies et d'immunothérapies anti-infectieuses par IVIG ou IVIG hyperimmunes.
Fc (Fc $\gamma$-récepteurs) des cellules du système réticulo-endothélial. L'administration des anticorps par voie topique au site même de développement de l'infection permet de contourner ces inconvénients [17, 18].

\section{Immunothérapie des infections respiratoires par administration topique d'anticorps}

L'immunothérapie passive des infections respiratoires par administration topique d'anticorps offre de nombreux avantages en termes d'efficacité et de tolérance $[5,17,18]$ (Tableau II). Le mécanisme d'action intervient aux étapes précoces de l'infection et impliquerait l'agglutination des microorganismes, empêchant l'inoculum infectieux de coloniser les épithéliums et facilitant son évacuation sous la forme d'agrégats dans le flux muco-ciliaire [19] (Figure 1). Ce mécanisme de neutralisation est efficace contre les pathogènes extracellulaires ou la dissémination intercellulaire des pathogènes intracellulaires, mais est inefficace contre les virus intracellulaires [20, 21]. Expérimentalement, la demi-vie des IVIG dans les fluides pulmonaires non inflammatoires de la souris est de 48 heures contre 7 jours dans le sang après injection intraveineuse, et les IVIG administrées par voie intranasale ne sont pas détectables dans le sang [16]. De plus, une partie des anticorps administrés peut subir une élimination par clairance mucociliaire et une protéolyse, ainsi qu'une capture non spécifique par les macrophages alvéolaires [22]. Néanmoins, les essais d'immunothérapie dans les pneumonies expérimentales par des anticorps délivrés par voie respiratoire ont démontré leur efficacité même à la phase de pneumonie constituée [20, 23, 24]. Des études expérimentales récentes ont montré que des IVIG ou des anticorps monoclonaux administrés par voie nasale neutralisaient des infections bactériennes et virales à des doses 10 à 100 fois plus faibles que les doses efficaces par 
voie veineuse. Cela est probablement dû au fait que la quantité nécessaire et suffisante d'anticorps neutralisants est délivrée au site même de développement du processus infectieux $[5,16-18,23,24]$. La neutralisation précoce de l'infection respiratoire est obtenue par des fragments $F(a b$ ')2 avec la même efficacité que les IgG complètes, suggérant que l'effet protecteur, indépendant de $\mathrm{Fc}$, ne requiert ni la fixation du complément ni l'intervention des phagocytes, et offre donc la possibilité de traiter des patients leucopéniques ou déficitaires en complément [4, 5, 912]. Cependant, l'efficacité d'une telle approche thérapeutique dans des conditions inflammatoires chez l'homme, comme l'asthme ou la mucoviscidose, n'a pas été évaluée. Les anticorps dirigés contre les pathogènes respiratoires, administrés par voie topique de manière prophylactique, sont efficaces en raison de leur action immédiate contre les étapes précoces de colonisation de l'épithélium et l'inhibition des effets cytotoxiques, mais leur action est moins évidente en thérapie curative de l'infection déclarée, du fait de l'importante charge infectieuse et des réactions inflammatoires de l'hôte.

\section{Immunothérapie des infections respiratoires par anticorps monoclonaux}

Les anticorps monoclonaux sont homogènes et spécifiques d'un épitope sélectionné. Ils sont habituellement produits in vitro, à partir de clones de lymphocytes $B$ de souris spécifiquement immunisées. Cependant, leur utilisation à des fins thérapeutiques chez l'homme reste limitée à des indications ne nécessitant pas d'administrations réitérées en raison de leur immunogénicité (réponse humaine anti-souris ou HAMA). Des anticorps monoclonaux obtenus par immortalisation de lymphocytes humains exprimant une immunoglobuline d'intérêt thérapeutique sont difficiles à produire. L'utilisation du génie génétique pour construire des anticorps humanisés est une solution élégante [25]. Des anticorps recombinants peuvent être produits par des bactéries, des levures, des cellules d'insectes et même des plantes transgéniques avec des coûts de production en baisse constante (de 1000 à 1 dollar le gramme pour un anticorps, selon sa production en culture de cellules de mammifères ou de plantes, respectivement [18]).

De nombreuses études sur des modèles cellulaires ou animaux ont permis de démontrer l'efficacité anti-infectieuse des anticorps monoclonaux [2, 5, 17, 18, 26]. Leur nature détermine également leur mode d'action. Des anticorps monoclonaux de classe IgA sont capables de neutraliser le virus Sendai [21] et le virus respiratoire syncytial (RSV) [27] par des mécanismes limités à l'agglutination et la neutralisation de l'attachement du pathogène à ses récepteurs, car les IgA n'activent pas le complément et ne se fixent pas aux récepteurs Fc $\gamma$ des phagocytes. Ils seraient cependant capables de neutraliser des pathogènes intracellulaires, par un mécanisme impliquant leur internalisation via leur attachement au récepteur pour les Ig polymériques [21]. Deux IgGl monoclonales humanisées reconnaissant la protéine de fusion du RSV neutralisent les deux sous-types du virus dans le modèle d'infection respiratoire chez le rat cotonnier [28]. Le plus efficace des deux, MEDI-493, a obtenu l'autorisation de mise sur le marché (palivizumab).

La spécificité des anticorps monoclonaux permet leur utilisation en tant que médicaments aux effets définis, mais forcément limités aux infections dont le diagnostic étiologique précis a été établi. Leur utilisation à titre prophylactique suppose que l'agent infectieux soit identifié, qu'il soit de nature clonale et qu'il exprime un épitope invariable. Tout variant antigénique échapperait ainsi à l'immunothérapie.

\section{Conclusions et perspectives}

L'avantage des immunoglobulines polyclonales sur les anticorps monoclonaux est de reconnaître un large spectre de variants antigéniques circulant parmi une population. Elles sont donc mieux adaptées à la prévention d'infections opportunistes dont l'étiologie est difficilement prédictible. Cependant, leur utilisation en tant que « médicament » antimicrobien pose des problèmes de reproductibilité et de standardisation de lot à lot. Les technologies de fabrication d'anticorps monoclonaux humanisés et de banques combinatoires d'anticorps offrent de réelles perspectives de reconstituer des mélanges d'anticorps, selon des conditions prédéterminées maîtrisant leur parfaite fonctionnalité $[18,25,26]$. L'ingénierie des anticorps est une approche innovante et les perspectives de développement industriel semblent prometteuses. Selon une récente étude, sept préparations thérapeutiques seraient en évaluation clinique chez six industriels différents [29]. L'amélioration constante de systèmes d'expression de protéines recombinantes assurant des rendements de synthèse élevés, une forte affinité et une parfaite glycosylation, devrait permettre d'élaborer de nouveaux anticorps anti-infectieux, efficaces et peu coûteux, pour lutter contre des maladies infectieuses, en particulier respiratoires, émergentes ou résistantes aux agents antimicrobiens. $\diamond$

- Activité immédiate
- Traitement ciblé au site même de l'infection
- Administration aisément reproductible et bien tolérée $[5,17,18]$
- N'interfère pas ou peu avec le système immunitaire de l'hôte $[2,5,18]$
- Permet d'adapter rapidement la préparation d'anticorps
à tout nouveau variant antigénique
- Limite le risque de sélection de variants résistants aux antibiotiques
- Immunisation active selon des modalités analogues à celles
de l'immunisation naturellement acquise [24]

Tableau II. Avantages de l'immunothérapie passive par anticorps administrés par voie topique. 


\section{SUMMARY}

Immunoprophylaxis of respiratory infections

Anti-infective antibody-based immunotherapy has gained renewed interest since the crisis of antibiotic resistance and because there is no therapy against various viral infections. The immunoprophylaxis of respiratory infections aims to utilize the ability of local antibodies to neutralize inhaled micro-organisms and their cytopathic products. Immunoglobulins for intravenous use (IVIG) have a wide spectrum of specificities. Hyperimmune IVIG containing high titers of specific antibodies have demonstrated efficacy in clinical trials, notably against the respiratory syncytial virus. Monoclonal antibodies have the advantage to be homogenous and specific for one selected epitope and several studies have demonstrated their efficacy to neutralize several infectious agents. Moreover, antibodies can be administered topically and are effective at lower doses than those needed for systemic administration. The mechanism of action could be the agglutination of bacteria or viruses at the epithelial surfaces of the respiratory tract inhibiting the early steps of the infectious process. Thanks to new technologies of humanized monoclonal antibodies, immunotherapy offers real promizing perspectives for prophylactic and therapeutic therapies against $a$ variety of current or emerging infectious diseases. $\diamond$

\section{RÉFÉRENCES}

1. Silverstein AM. History of immunology. In: Paul WE, ed. Fundamental immunology. New York: Raven Press, 1984: 23-40.

2. Casadevall A, Scharff MD. Return to the past : the case for antibody-based therapies in infectious diseases. Clin Infect Dis 1995; 21 : 150-61.

3. Lister PD. Emerging resistance problems among respiratory tract pathogens. Am J Manag Care 2000; 6: S409-18.

4. Busse PJ, Razvi S, Cunningham-Rundles C. Efficacy of intravenous immunoglobulin in the prevention of pneumonia in patients with common variable immunodeficiency. J Allergy Clin Immunol 2002; 109: 1001-4.

5. Hammarström L, Gardulf A, Hammarström V, et al. Systemic and topical immunoglobulin treatment in immunocompromised patients. Immunol Rev 1994; 139: 43-70.

6. Enarson DA, Chretien J. Epidemiology of respiratory infectious diseases. Curr Opin Pulm Med 1999; 5 : 128-35.

7. Reynolds Hy. Normal and defective respiratory host defenses. In: Pennington JE, ed. Respiratory infections. New York : Raven Press, 1994: 1-33.

8. Lamari F, Karamanos NK, Papadopoulou-Alataki $\varepsilon$, et al. Monitoring of two intravenous immunoglobulin. Preparations for immunoglobulin $G$ subclasses and specific antibodies to bacterial surface antigens and relation with their levels in treated immunodeficient patients. J Pharm Biomed Anal 2000; 22: 1029-36.

9. Groothuis JR, Simoes EA, Levin MJ, et al. Prophylactic administration of respiratory syncytial virus immune globulin to high-risk infants and young children. The respiratory syncytial virus immune globulin study group. N EnglJ Med 1993; 329: $1524-30$.
10. Baker JC, Melish ME, Hall RT, et al. Intravenous immune globulin for the prevention of nosocomial infection in low-birth-weight neonates. The multicenter group for the study of immune globulin in neonates. N Engl J Med 1992; 327 : 213-9.

11. Fanaroff $A A$, Korones $S B$, Wright $L L$, et al. A controlled trial of intravenous immune globulin to reduce nosocomial infections in very-low-birth-weight infants. National institute of child health and human development neonatal research network. N Engl J Med 1994; 330: 1107-13.

12. Spector SA, Gelber RD, McGrath N, et al. A controlled trial of intravenous immune globulin for the prevention of serious bacterial infections in children receiving zidovudine for advanced human immunodeficiency virus infection. Pediatric AIDS clinical trials group. $N$ Engl J Med 1994; 331 : 1181-7.

13. Douzinas $\varepsilon \varepsilon$, Pitaridis MT, Louris G, et al. Prevention of infection in multiple trauma patients by high dose intravenous immunoglobulins. Crit Care Med 2000; 28: 8-15.

14. Conti DJ, Freed BM, Gruber SA, Lempert N. Prophylaxis of primary cytomegalovirus disease in renal transplant recipients. A trial of ganciclovir vs immunoglobulin. Arch Surg 1994; 129: 443-7.

15. Granström M, Olinder-Nielsen AM, Holmblad P, et al. Specific immunoglobulin for treatment of whooping cough. Lancet 1991; 338: 1230-3.

16. De Hennezel L, Ramisse F, Binder $P$, et al. Effective combination therapy for invasive pneumococcal pneumonia with ampicillin and intravenous immunoglobulins in a mouse model. Antimicrob Agents Chemother 2001; 45: 316-8.

17. Weltzin R, Monath TP. Intranasal antibody prophylaxis for protection against viral disease. Clin Microbiol Rev 1999; 12: 383-93.

18. Zeitlin L, Cone RA, Whaley KJ. Using monoclonal antibodies to prevent mucosal transmission of epidemic infectious diseases. Emerg Infect Dis 1999; 5: 54-64.

19. Ma JK, Hunjan M, Smith R, et al. An investigation into the mechanism of protection by local passive immunization with monoclonal antibodies against Streptococcus mutans. Infect Immun 1990; 58: 3407-14.

20. Ramisse F, Deramoudt FX, Szatanik M, et al. Effective prophylaxis of influenza $A$ virus pneumonia in mice by topical passive immunotherapy with polyvalent human immunoglobulins or $\mathrm{F}\left(\mathrm{ab}^{\prime}\right) 2$ fragments. Clin Exp Immunol 1998; 111: 583-7.

21. Mazanec MB, Kaetzel CS, Lamm ME, et al. Intracellular neutralization of virus by immunoglobulin A antibodies. Proc Natl Acad Sci USA 1992; 89: 6901-5.

22. Lombry C, Edwards DA, Preat V, Vanbever R. Alveolar macrophages are a primary barrier to pulmonary absorption of macromolecules. Am J Physiol Lung Cell Mol Physiol 2004; 286: L1002-8.

23. Ramisse F, Szatanik M, Binder P, Alonso JM. Passive local immunotherapy of experimental staphylococcal pneumonia with human intravenous immunoglobulin. J Infect Dis 1993; 168: 1030-3.

24. Ramisse F, Binder P, Szatanik M, Alonso JM. Passive and active immunotherapy of experimental pneumococcal pneumonia by polyvalent human immunoglobulin or $F\left(a b^{\prime}\right) 2$ fragments administered intranasally. J Infect Dis 1996; 173: 1123-8.

25. Winter G, Griffiths AD, Hawkins RE, Hoogenboom HR. Making antibodies by phage display technology. Ann Rev Immunol 1994; 12: 433-55.

26. Breedveld FC. Therapeutic monoclonal antibodies. Lancet 2000; 355: 735-40.

27. Weltzin R, Traina-Dorge V, Soike K, et al. Intranasal monoclonal IgA antibody to respiratory syncytial virus protects rhesus monkeys against upper and lower respiratory tract infection. J Infect Dis 1996; 174: 256-61.

28. Johnson S, Griego SD, Pfarr DS, et al. A direct comparison of the activities of two humanized respiratory syncytial virus monoclonal antibodies: MEDI-493 and RSHZ19. J Infect Dis 1999; 180: 35-40.

29. Gavilondo JV, Larrick JW. Antibody engineering at the millenium. Biotechniques $2000 ; 29: 128-45$.

TIRÉS À PART

C. Dreffier

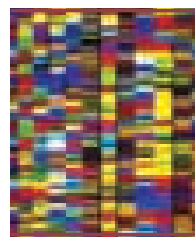

\section{Retrouvez chaque mois médecine/sciences sur France-Info dans la chronique «Info-Sciences 》 de Marie-Odile Monchicourt, du lundi au mercredi. france-info.com}

